\title{
Fusion-based Sensing for Uncertain Primary User Signal in Cognitive Ratio Networks
}

\author{
Tai-Lin Chin and Cheng-Chia Huang \\ Department of Computer Science and Information Engineering \\ National Taiwan University of Science and Technology \\ Taipei, Taiwan 106 \\ Email: tchin@mail.ntust.edu.tw
}

\begin{abstract}
In cognitive radio networks, secondary users can use the licensed frequency bands if the primary user is idle. In order to protect the privilege of the primary user, detecting the presence of the primary user in cognitive radio networks is important. Collaborative sensing is a possible method to improve the detection performance for the presence of the primary user. The IEEE 802.22 standard provides an appropriate topology to perform collaborative sensing. This paper addresses the problem of collaborative sensing in cognitive radio networks. The proposed sensing mechanism is not based on any known signal or noise distributions. The approximations of the distributions for the signal energy measurements are first derived. The SUM fusion rule is adopted to make the final consensus about the presence of the primary user. Based on the derived measurement distributions, detection performance in terms of detection probability subject to a fixed false alarm probability is evaluated analytically. The analytic results of the measurement distributions and the detection performance are very close to the simulation results. In particular, it is found that adding more users to participate in the fusion does not improve the detection performance. In fact, it may hurt the detection performance. A better strategy is to include only a few users with the highest SNR in the fusion. In this way, the detection performance is higher while communication costs can be reduced.
\end{abstract}

\section{INTRODUCTION}

Cognitive radio technology allows unlicensed (secondary) users to reuse frequency bands when licensed (primary) users are not using the bands. It provides a potential solution to increase the utilization of the current scarce frequency band resources. To protect the privilege of the licensed users, unlicensed users have to sense the presence of the licensed users and vacate the channel if the licensed users are detected. Thus, spectrum sensing is an important problem to realize cognitive radio technology [1]. Collaborative sensing is a possible method to improve the detection performance. One the one hand, it provides better protection to the primary users. On the other hand, it can increases the utilization of the channel for the secondary users.

The IEEE 802.22 Wireless Regional Area Network (WRAN) standard specifies the protocol to reuse the spectrum allocated to TV broadcast service using cognitive radio techniques [2]. The general topology of the network is shown in Fig. 1. Essentially, the network contains a TV broadcast station, i.e., the primary user, a secondary user base station (BS), and customer-premises equipments (CPE), i.e., the secondary users. The CPEs will be sensing the spectrum and periodically send their sensing reports to the BS. The BS then decides

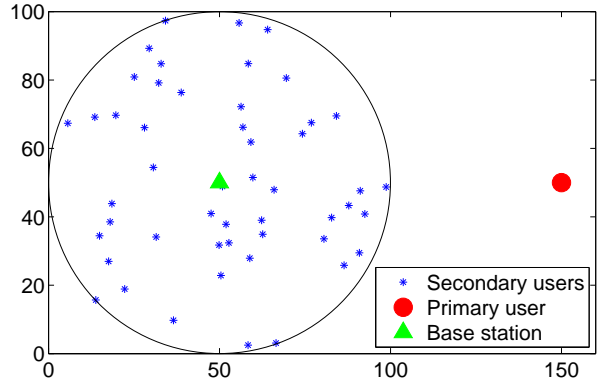

Fig. 1. Deployment topology of the network

whether to change or stay in the currently used channel and informs the CPEs. The scenario creates a great opportunity to perform collaborative sensing where the BS can be the fusion center and fuse the collected reports to arrive at a consensus decision about the presence of the primary user.

This paper investigates collaborative sensing for the presence of uncertain primary user signal based on the IEEE802.22 topology. Generally, each CPE periodically reports to the BS its local decision about the presence or absence of the primary user. To reserve energy, the CPEs only need to report a one bit decision based on their local observations. The BS then fuses the collected local decisions to arrive at a consensus. If a positive decision about the presence of the primary user is made, the BS informs the CPEs to change channel or stop the current transmission. Two metrics are important to evaluate the performance of the sensing operation, namely the detection probability and the false alarm probability. The detection probability is defined as the probability that the detector declares a primary user is present while, indeed, there is a primary user present. In contrast, the false alarm probability is defined as the probability that the detector declares a primary user is present while actually no primary user is present. The detection probability provides a metric to judge the protection level of the primary user's privilege and the false alarm probability can evaluate the level of secondary user utilization. Basically, a desired detector should have the characteristics that the detection probability is as high as possible while the false alarm probability is low. Collaborative sensing can improve the detection probability while reducing the false alarm probability.

In this paper, constant false alarm rate (CFAR) detector is 
adopted for making both the local decision at each CPE and the global decision at the BS. To control a constant false alarm rate and to evaluate the detection probability, the distributions of the signal measurements have to be determined. Unlike many previous studies, this paper does not make any prior assumptions about the distributions of the monitored signal and noise. Thus, the distributions of the signal energy measurements are first estimated. The detection performance in terms of the detection probability subject to a fixed false alarm probability is analyzed.

Extensive simulations are conducted to verify the correctness of the analyses for the detection performance. The detection performance of the proposed SUM fusion rule is also compared to those of the conventional used AND fusion and OR fusion rules [3]. The SUM fusion outperforms AND and OR fusion in detection probability based on a constraint of a fixed false alarm rate. In particular, it is shown that having all the secondary users to participate in the fusion may not be beneficial to the detection performance. In fact, the detection probability may be decreased if too many users participate in the fusion. To include a small number of users who have the highest signal to noise ratio in the fusion can perform better than include all.

The paper is organized as follows. Section II reviews the related work. Section III describes the preliminary assumptions and basic analysis of the signal energy measurements. Section IV presents the performance evaluations of the collaborative sensing technique. Section V shows the simulation results. The paper concludes in Section VI.

\section{RELATED WORK}

It has been shown that most of the licensed spectrum is surprisingly under-utilized [4]. Cognitive radio provides a potential mechanism to increase the utilization of the limited spectrum. One of the most important problems in cognitive radio is spectrum sensing. In general, spectrum sensing methods can be separated in two categories, i.e., energy detection [5], and feature detection [6], [7]. Energy detection is based on checking the likelihood of the observed signal occurring in two hypotheses which assume the conditions of the presence and absence of the target signal. If the probability density functions (PDF) under both hypotheses are perfectly known, energy detector has been shown to be close to the optimal detector [6], [8]. In [8], it has been shown that the performance of energy detector is asymptotically same as the optimal detector when the signal to noise ratio is low based on the assumptions that the signals are modulated with a zero-mean finite signal constellation and the PDFs are perfectly known as well as symbols are independent. Similar results are presented for detecting an OFDM signal in [6]. However, a fundamental limit for energy detection is the SNR wall problem when the SNR is relatively small [9], [10]. The detection performance of the energy detector may deteriorate if the noise variance is not perfectly known [11]. Moreover, if the noise variance is unknown, energy detector cannot be used since the it is required to set the detection threshold.

Signal used in practical communications usually contains certain features. For example, OFDM modulation adds a cyclic prefix $(\mathrm{CP})$. Some other systems multiplex known pilots into the transmitted data or superimpose pilot signal on the transmitted signals. Feature detection employs the distinctive features to discriminate the presence of the primary user [12], [13]. Two typical methods for feature detection is the autocorrelation-based detector [12] and the cyclostationarity detector [14]. These detectors differentiate the primary user signal from the local noise signal by exploiting certain periodical features shown in the mean and autocorrelation of a signal based on a particular modulation. When the parameters like the noise power and signal power are not known, feature detection could outperform energy detection [15].

It is inevitable to increase the interference of primary users in cognitive radio, since reliable sensing is not always guaranteed because of the impact of multipath fading, shadowing, and other factors. Consequently, a variety of studies have focused on cooperative spectrum sensing to provide reliable detection of the presence of the primary users [16]-[19]. In [20], it is shown that it may not be beneficial to involve all secondary users in the cooperation due to the different locations and channel conditions of the secondary users. Consequently, the optimal secondary user selection for cooperative sensing is also investigated in [21]. Recently, there is also research studying the security problems for cooperative sensing in cognitive radio [22], [23] or power control for coordinating spectrum sharing [24]. However, the problem we consider differs in that it is restricted to detect a known primary user signal. In fact, the primary user signal can be in any distributions. Further, it considers different fusion methods to improve the detection performance.

\section{PRELIMINARY FOUNDATION}

\section{A. Sensing hypotheses}

To model the signal received by a secondary user, let $S(t)$ denote the signal emitted from the primary user at time instant $t$. Assume that $S(t)$ is an i.i.d. random process with mean $\mu_{s}$ and $\sigma_{s}$. Let $d_{i}$ be the distance from the primary user to secondary user $i$. The primary user signal received by user $i$ at a certain time instant $t$ can be modeled as follows:

$$
S_{i}(t)=\frac{S(t)}{d_{i}^{\alpha}},
$$

where $\alpha$ is the path loss exponent. Obviously, the mean and variance of $S_{i}(t)$ is given by

$$
\mu_{s, i}=\frac{\mu_{s}}{d_{i}^{\alpha}} \text { and } \sigma_{s, i}^{2}=\frac{\sigma_{s}^{2}}{d_{i}^{2 \alpha}},
$$

respectively. It is noted that the distribution of the signal is assumed to be unknown in this paper. However, it is relatively easy to estimate the mean and variance of the signal by users. In addition, the received signal is usually corrupted by noise.

In cognitive radio networks, since the primary user may be absent, there are two hypotheses for the received signal. Let $\mathcal{H}_{0}$ denote the null hypothesis that the primary user is absent and $\mathcal{H}_{1}$ denote the alternative hypothesis that the primary user is present. The received signal of user $i$ can be expressed as follows:

$$
\begin{array}{ll}
\mathcal{H}_{1}: & y_{i}(t)=S_{i}(t)+X_{i}(t), \text { and } \\
\mathcal{H}_{0}: & y_{i}(t)=X_{i}(t),
\end{array}
$$


where $X_{i}(t)$ denotes the noise, which is assumed to have mean $\mu_{x, i}$ and variance $\sigma_{x, i}$.

\section{B. Measurement PDF estimation}

Energy detector is usually exploited to sense the presence or absence of a certain signal. The detector periodically collects the signal energy, which is given by the following statistics:

$$
M_{i}=\frac{1}{T} \sum_{t=1}^{T}\left|y_{i}(t)\right|^{2}
$$

However, the probability distribution of the signal energy measurement is critical for making detection decisions. Since the probability distribution of the signal is assumed to be unknown, the probability distribution of the measurements is first evaluated by approximation.

Under the hypothesis $\mathcal{H}_{0}$, the measurements contain only noise, i.e.,

$$
M_{i}=\frac{1}{T} \sum_{t=1}^{T}\left|X_{i}(t)\right|^{2}
$$

Assume that $X_{i}(t)$ is i.i.d.. For large $T$, the distribution of $M_{i}$ can be approximated by Central Limit Theorem (CLT) if the mean and variance of $X_{i}(t)^{2}$ can be determined. It is easy to obtain the mean of $X_{i}(t)^{2}$ is $\mu_{x, i}^{2}+\sigma_{x, i}^{2}$. However, no close form expression can be derived for the variance. Fortunately, the approximation of the variance of $X_{i}(t)^{2}$ can be obtained by Delta method [25], which is described in Proposition 1.

Proposition 1: (Delta method) Let $x$ be a random variable with mean $\mu$ and variance $\sigma^{2}$. The variance of a function $f(x)$ can be approximated by

$$
\operatorname{Var}(f(x)) \approx\left[f^{\prime}(\mu)\right]^{2} \times \sigma^{2} .
$$

Assume $f\left(X_{i}(t)\right)=X_{i}(t)^{2}$. From Proposition 1, the variance of $X_{i}(t)^{2}$ can be approximated by $4 \mu_{x, i}^{2} \sigma_{x, i}^{2}$. Thus, using CLT, the probability density function (PDF) of $M_{i}$ converges in distribution to a Gaussian distribution with the mean and variance as follows:

$$
\begin{aligned}
\text { mean: } & \mu_{i, 0}=\mu_{x, i}^{2}+\sigma_{x, i}^{2} \\
\text { variance: } & \sigma_{i, 0}^{2}=\frac{4}{T} \mu_{x, i}^{2} \sigma_{x, i}^{2} .
\end{aligned}
$$

Under the hypothesis $\mathcal{H}_{1}$, the energy measurement statistics are given by

$$
M_{i}=\frac{1}{T} \sum_{t=1}^{T}\left|y_{i}(t)\right|^{2}=\frac{1}{T} \sum_{t=1}^{T}\left|S_{i}(t)+X_{i}(t)\right|^{2}
$$

To evaluate the PDF of $M_{i}$, eq.(9) is decomposed to three terms as follows:

$$
M_{i}=\frac{1}{T} \sum_{t=1}^{T}\left|S_{i}(t)\right|^{2}+\frac{1}{T} \sum_{t=1}^{T}\left|X_{i}(t)\right|^{2}+\frac{2}{T} \sum_{t=1}^{T}\left|S_{i}(t) X_{i}(t)\right|
$$

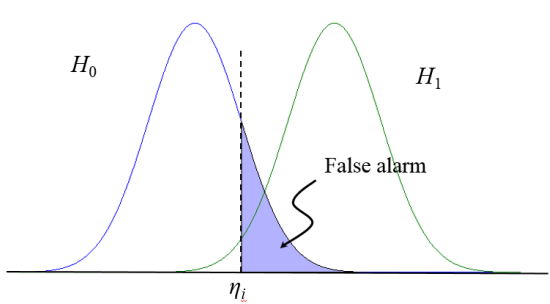

Fig. 2. Constant false alarm rate detection

Similarly, the first two terms in eq.(10) can be approximated by CLT and proposition 1 . Thus,

$$
\begin{array}{r}
\frac{1}{T} \sum_{t=1}^{T}\left|S_{i}(t)\right|^{2} \sim N\left(\mu_{s, i}^{2}+\sigma_{s, i}^{2}, \frac{4}{T} \mu_{s, i}^{2} \sigma_{s, i}^{2}\right) \\
\frac{1}{T} \sum_{t=1}^{T}\left|X_{i}(t)\right|^{2} \sim N\left(\mu_{x, i}^{2}+\sigma_{x, i}^{2}, \frac{4}{T} \mu_{x, i}^{2} \sigma_{x, i}^{2}\right)
\end{array}
$$

For the third term in eq.(10), since $S_{i}(t)$ and $X_{i}(t)$ are independent, the mean of $S_{i}(t) X_{i}(t)$ can be derived as

$$
E\left[S_{i}(t) X_{i}(t)\right]=E\left[S_{i}(t)\right] E\left[X_{i}(t)\right]=\mu_{s, i} \mu_{x, i},
$$

and the variance can be obtained by

$$
\begin{aligned}
\operatorname{Var}\left(S_{i}(t) X_{i}(t)\right) & =E\left[\left(S_{i}(t) X_{i}(t)-\mu_{s, i} \mu_{x, i}\right)^{2}\right] \\
& =E\left[S_{i}(t)^{2}\right] E\left[X_{i}(t)^{2}\right]-\mu_{s, i}^{2} \mu_{x}^{2}(15) \\
& =\mu_{s, i}^{2} \sigma_{x, i}^{2}+\mu_{x, i}^{2} \sigma_{s, i}^{2}+\sigma_{s, i}^{2} \sigma_{x, i}^{2} .
\end{aligned}
$$

Again, by CLT, the PDF of the third term of eq.(10) is given by

$$
\begin{aligned}
& \frac{2}{T} \sum_{t=1}^{T} S_{i}(t) X_{i}(t) \sim \\
& N\left(2 \mu_{s, i} \mu_{x, i}, \frac{4}{T}\left(\mu_{s, i}^{2} \sigma_{x, i}^{2}+\mu_{x, i}^{2} \sigma_{s, i}^{2}+\sigma_{s, i}^{2} \sigma_{x, i}^{2}\right)\right) .
\end{aligned}
$$

Obviously, one can add the three Gaussian distribution in eq.(11), eq.(12), and eq.(18) to get the PDF of the measurement $M_{i}$. Thus, under the hypothesis $\mathcal{H}_{1}$, the PDF of $M_{i}$ can be approximated by a Gaussian distribution $N\left(\mu_{i, 1}, \sigma_{i, 1}^{2}\right)$, where $\mu_{i, 1}$ and $\sigma_{i, 1}^{2}$ are as follows:

$$
\begin{aligned}
\mu_{i, 1} & =\sigma_{s, i}^{2}+\sigma_{x, i}^{2}+\left(\mu_{s, i}+\mu_{x, i}\right)^{2} \\
\sigma_{i, 1}^{2} & =\frac{4}{T}\left(\mu_{s, i}^{2}+\mu_{x, i}^{2}\right)\left(\sigma_{s, i}^{2}+\sigma_{x, i}^{2}\right)+\sigma_{s, i}^{2} \sigma_{x, i}^{2}
\end{aligned}
$$

Note that the mean in eq.(7) and eq.(19) are exact, but the variance in eq. (8 and eq.(20) are estimated by Proposition 1.

\section{COLlaborative SENSING}

\section{A. Local detction}

For collaborative sensing, the secondary users first make their own local decisions about the presence of the primary user. Then, report the local decision to the BS. With the probability distributions of the measurements under the two 
hypotheses, constant false alarm rate (CFAR) detector is one of the potential detectors which can be used to make binary decision between the two hypotheses. As shown in Fig. 2, the CFAR detector uses a threshold $\eta_{i}$ to detect anomaly measurements. When the measurement $M_{i}$ is greater than $\eta_{i}$, it declares that the primary user is present. Otherwise, it decides that the primary user is absent. False alarm is defined as the cases that the detector declares a primary user present when the primary user is actually absent.

Consequently, given the PDF of the measurements under $\mathcal{H}_{0}$ and a tolerable false alarm probability, one can determine the threshold $\eta_{i}$. Formally, the false alarm probability can be derived as

$$
\begin{aligned}
P_{f, i} & =P\left(M_{i}>\eta_{i} \mid H_{0}\right) \\
& =Q\left(\frac{\eta_{i}-\mu_{i, 0}}{\sigma_{i, 0}}\right),
\end{aligned}
$$

where $Q$ is the tail probability of a standard normal distribution, i.e.,

$$
Q(x)=\frac{1}{\sqrt{2 \pi}} \int_{x}^{\infty} \exp \left(-\frac{u^{2}}{2}\right) d u .
$$

Recall that $\mu_{i, 0}$ and $\sigma_{i, 0}$ are the mean and standard deviation estimated for the PDF of the measurement under $\mathcal{H}_{0}$ in eq.(7) and eq.(8).

In contrast, detection probability is defined as the probability that the detector declares a primary user present when there is actually a primary user present. Therefore, with the threshold $\eta_{i}$ obtained from the above constraint of false alarm probability, the detection probability can be derived by

$$
\begin{aligned}
P_{d, i} & =P\left(M_{i}>\eta_{i} \mid H_{1}\right) \\
& =Q\left(\frac{\eta_{i}-\mu_{i, 1}}{\sigma_{i, 1}}\right),
\end{aligned}
$$

where $\mu_{i, 1}$ and $\sigma_{i, 1}$ are from eq.(19) and eq.(20), respectively.

Note that in case the detection probability is more important than the false alarm probability, one can also determine the threshold $\eta_{i}$ by specifying a desired constraint on detection probability and then derive the resulting false alarm probability. In this way, the detection performance in terms of detection probability is guaranteed.

\section{B. Global decision making}

The BS collects the local decisions from secondary users and fuse the local decisions to arrive at a consensus. Denote the binary decision from secondary user $i$ as $I_{i}=0,1$. An intuitive fusion method is to count the number of positive decisions as in eq.(25). If the total number of positive decisions is greater than a threshold $\lambda$, it declares that a primary user is present. Otherwise, it decides that the primary user is absent.

$$
\Lambda=\sum_{i=1}^{n} I_{i} \underset{H_{0}}{\stackrel{H_{1}}{\gtrless}} \lambda
$$

CFAR detector can also be used as the detector for global decision making. However, to determine an appropriate threshold $\lambda$ subject to a fixed global false alarm probability constraint, the PDF of $\Lambda=\sum_{i=1}^{n} I_{i}$ under $\mathcal{H}_{0}$ is required.
Analogously, given the threshold $\lambda$, the PDF of $\Lambda$ under $\mathcal{H}_{1}$ must be available to evaluate the detection probability.

Obviously, $I_{i}$ follows follows Bernoulli distribution with $P_{f, i}\left(P_{d, i}\right)$ as the probability of success under the condition $\mathcal{H}_{0}\left(\mathcal{H}_{1}\right)$. However, each secondary user is not necessary to set the same local false alarm probability as the constraint. In addition, since the detection probability is highly dependent on the distance from the primary user to the secondary user, it is highly possible that the local detection probability is different at different users. Consequently, the total number of detections $(\Lambda)$ does not follow a Binomial distribution. It is very complicated and difficult to get the exact expression for the distributions of $\Lambda$. Thus, the approximations of the distributions are derived.

Note that $\left\{I_{1}, \ldots, I_{n}\right\}$ are mutually independent. According to CLT as well as Lyapunov condition [26], when $n$ is large, the distribution of $\Lambda$ converges to a Gaussian distribution with mean $\mu$ and variance $\sigma^{2}$, where

$$
\begin{aligned}
\mu & =E[\Lambda]=\sum_{i=1}^{n} E\left[I_{i}\right], \text { and } \\
\sigma^{2} & =\operatorname{var}[\Lambda]=\sum_{i=1}^{n} \operatorname{var}\left[I_{i}\right] .
\end{aligned}
$$

It is easy to see that under $\mathcal{H}_{0}, E\left[I_{i}\right]=P_{f, i}$ and $\operatorname{var}\left[I_{i}\right]=$ $P_{f, i}\left(1-P_{f, i}\right)$. Therefore, $\Lambda$ converges to a Gaussian distribution with mean $\mu_{0}$ and variance $\sigma_{0}^{2}$, where

$$
\mu_{0}=\sum_{i=1}^{n} P_{f, i} \text { and } \sigma_{0}^{2}=\sum_{i=1}^{n} P_{f, i}\left(1-P_{f, i}\right) .
$$

Analogously, under $\mathcal{H}_{1}, E\left[I_{i}\right]=P_{d, i}$ and $\operatorname{var}\left[I_{i}\right]=$ $P_{d, i}\left(1-P_{d, i}\right)$. Thus, $\Lambda$ converges to a Gaussian distribution with mean $\mu_{1}$ and variance $\sigma_{1}^{2}$, where

$$
\mu_{1}=\sum_{i=1}^{n} P_{d, i} \text { and } \sigma_{1}^{2}=\sum_{i=1}^{n} P_{d, i}\left(1-P_{d, i}\right) .
$$

The global false alarm probability can then be derived as

$$
P_{f}=P\left(\Lambda \geq \lambda \mid H_{0}\right)=Q\left(\frac{\lambda-\mu_{0}}{\sigma_{0}}\right) .
$$

Given a global false alarm probability $p_{f}$, one can determine the threshold $\lambda$ as follows:

$$
\lambda=\left\lceil Q^{-1}\left(p_{f}\right) \times \sigma_{0}+\mu_{0}\right\rceil .
$$

Note that $\lambda$ is an integer threshold for the number of users who report a positive detect. Then, the global detection probability can be evaluated as

$$
P_{d}=P\left(\Lambda \geq \lambda \mid H_{1}\right)=Q\left(\frac{\lambda-\mu_{1}}{\sigma_{1}}\right) .
$$

In particular, if all the users set the same local false alarm probability, i.e., let $P_{f, i}=p_{f}, \forall i$, the exact global false alarm probability can be derived as

$$
P_{f}=\sum_{i=\lambda}^{n}\left(\begin{array}{c}
n \\
i
\end{array}\right) p_{f}^{i}\left(1-p_{f}^{i}\right)^{n-i}
$$


$P_{f}$ follows Binomial distribution. When $n$ is large, eq.(33) can be approximated by Laplace-DeMoivre approximation as follows:

$$
P_{f} \simeq Q\left(\frac{\lambda-n p_{f}}{\sqrt{n p_{f}\left(1-p_{f}\right)}}\right),
$$

which is the same as in eq.(30).

\section{Simulations}

In the simulations, the correctness and performance of the addressed collaborative sensing method are investigated. The simulations are conducted in a topology as shown in Fig. 1. There 50 secondary users who are uniformly distributed within the $50 \mathrm{~km}$ radius region of the BS. The users cooperates to sense the presence of a signal from the primary user, who is $100 \mathrm{~km}$ away from the BS. The signal strength, $S(t)$, of the primary user is assume to be a random variable with mean 200 and variance 3 . The path loss exponent $\alpha$ is set to be 2 . The noise process at each individual user is randomly set to have mean 1 to 3 and variance between 0 to 1 . If no further described, the local false alarm probability is set to be 0.2 for each individual user and the number of samples $T$ used in the energy measurement in eq.(5) is set to be 50. Without loss of generality, Gaussian random process is used to generate the signal and noise. However, the signal and noise can follow any other processes without affecting the correctness of the results.

The distributions of energy measurements $M_{i}$ are first investigated. Note that the derived means of $M_{i}$ in eq.(7) and eq.(19 are exact, but the variance in eq.(8 and eq.(20) are approximated. Since the signal and noise are generated by Gaussian distribution, the true variance of $M_{i}$ can be derived. Let $x$ be a random variable with mean $\mu_{x}$ and variance $\sigma_{x}$. Assume that

$$
y=\left(\frac{x-\mu_{x}}{\sigma_{x}}\right) .
$$

Taking the square and rearranging the equation, we can get

$$
\sigma_{x}^{2} y^{2}=x^{2}-2 \mu_{x} x+\mu_{x}^{2} .
$$

Taking the variance of both sides, one can get

$$
\operatorname{var}\left(\sigma_{x}^{2} y^{2}\right)=\operatorname{var}\left(x^{2}\right)-\operatorname{var}\left(2 \mu_{x} x\right) .
$$

Since $y^{2}$ is a random variable of Chi-square with one degree of freedom, the variance of $y^{2}$ is 2 . Therefore,

$$
\operatorname{var}\left(x^{2}\right)=2 \sigma_{x}^{4}+4 \mu_{x}^{2} \sigma_{x}^{2} .
$$

Finally, the variance of $\frac{1}{T} \sum_{t=1}^{T} x^{2}$ is

$$
\frac{1}{T}\left(2 \sigma_{x}^{4}+4 \mu_{x}^{2} \sigma_{x}^{2}\right) \text {. }
$$

Thus, let $x$ be the noise, the variance of $M_{i}$ under $\mathcal{H}_{0}$ can be obtained. Analogously, let $x$ be the primary user signal plus noise, one can calculate the variance of $M_{i}$ under $\mathcal{H}_{1}$.

The user at location $(98.7,48.7)$ is chosen as an example to show the statistics of the sampled energy measurements. The noise process at this user is assumed to have mean 1.5654 and variance 0.2835 . Table I shows the statistics of the mean and variance of the energy measurement $M_{i}$. The estimated variances are very close to the true values. Fig. 3 shows the corresponding histograms of the collected statistics and the
TABLE I. MEASUREMENT STATISTIC CHARACTERISTICS OF A USER AT $(98.7,48.7)$

\begin{tabular}{|l|l|l||l|l|l|}
\hline$H_{0}$ & \multicolumn{2}{|c||}{ Mean } & \multicolumn{3}{c|}{ Variance } \\
\hline$T$ & Simulation & True & Simulation & Estimation & True \\
\hline 10 & 2.517312 & 2.530849 & 0.083116 & 0.078780 & 0.080072 \\
\hline 50 & 2.528358 & 2.530849 & 0.016317 & 0.015756 & 0.016014 \\
\hline 100 & 2.529594 & 2.530849 & 0.007571 & 0.007878 & 0.008007 \\
\hline \hline$H_{1}$ & \multicolumn{2}{|c||}{ Mean } & \multicolumn{3}{c|}{ Variance } \\
\hline$T$ & Simulation & True & Simulation & Estimation & True \\
\hline 10 & 2.760625 & 2.774720 & 0.091265 & 0.078967 & 0.087914 \\
\hline 50 & 2.772109 & 2.774720 & 0.017906 & 0.015793 & 0.017583 \\
\hline 100 & 2.773406 & 2.774720 & 0.008317 & 0.007897 & 0.008791 \\
\hline
\end{tabular}

estimated pdf of the energy measurements. From the figures, when $T=10$, the distributions of the energy measurements under $\mathcal{H}_{0}$ and $\mathcal{H}_{0}$ overlap over a large area. This implies that the CPE may be difficult to differentiate the measurements with and without the presence of the primary user. However, when $T$ increases, the variance of the measurements decreases, and the distributions of the measurements under $\mathcal{H}_{0}$ and $\mathcal{H}_{0}$ are separated more obviously. Thus, the CPE can have higher local detection probability when $T$ is larger, given a certain false alarm probability constraint.

Fig. 4 shows the ROC curves for the collaborative sensing operations with different number of samples used in evaluating the energy measurements. The local false alarm probability is set to be 0.2 for each user and the detection threshold $\eta_{i}$ is calculated by eq.(22). The users report their local decisions to the BS where the consensus is arrived. The global detector uses eq.(31) to determine the global detection threshold. Each data point in the figure is the result from 1000 runs of the detection operations. From the figure, the estimated results are very close to the simulated results. The small discrepancy is resulting from the approximation when evaluating the variance of the energy measurements and the application of CLT to evaluate the distributions of the energy measurements under the hypotheses $\mathcal{H}_{0}$ and $\mathcal{H}_{1}$. In addition, the detection performance is better if the number of samples, $T$, used to evaluate the average energy measurement in eq.(5) is large. This is because when $T$ is large, the distributions of energy measurements under $\mathcal{H}_{1}$ and $\mathcal{H}_{0}$ are separated more clearly with less overlap as shown in Fig.3. Therefore, secondary users can be much easier to tell the presence of the primary with higher probability. In other words, the local detection probability is higher. From eq. (29, the mean of the sum of local decision $\mu_{1}$ would become larger and the variance may not be affected too much. Thus, the global detection probability in eq.(32) will increase as $T$ increases.

Fig. 5 shows the number of users participating in the collaboration versus the global detection probability. The users are ordered according to the signal to noise ratio. In the simulations, if $k$ users participate in the collaboration, only the $k$ users with the top $k$ highest SNR will participate in making the final decision. The results look serrated because the final decision threshold in eq.(31) is taken only an integer. Although it is serrated, one can still determine that all users participate in the collaboration may not be helpful for improving the detection performance. When the number of users participating in the collaboration is very small, for example less than 10 , adding users in the collaboration could improve the detection performance. However, adding more users will not improve 


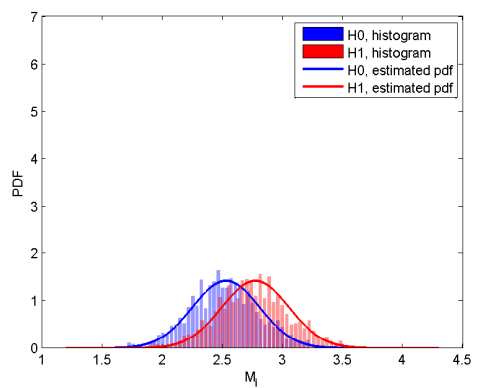

(a) $\mathrm{T}=10$

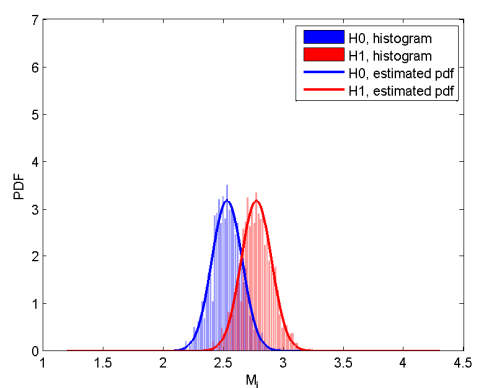

(b) $\mathrm{T}=50$

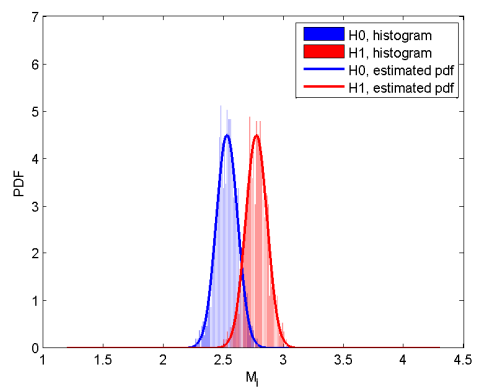

(c) $\mathrm{T}=100$

Fig. 3. The histograms of energy measurements

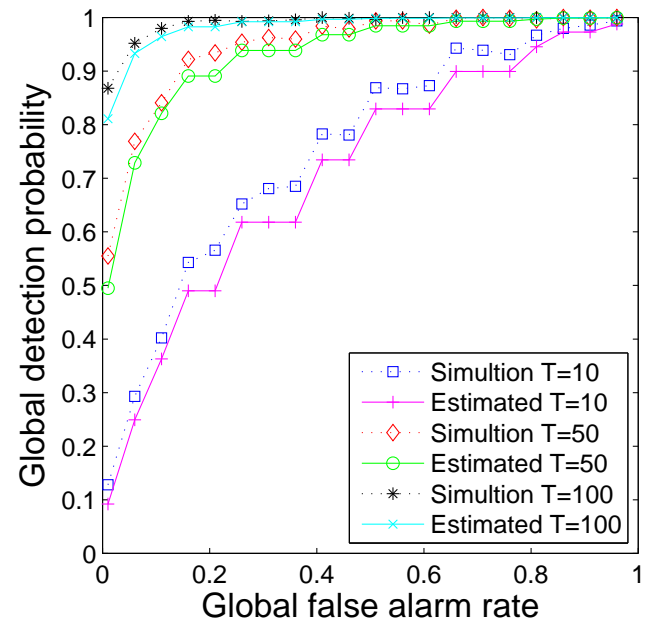

Fig. 4. The ROC curves with different $T$ values

the detection performance further. It is suggested that it is enough to choose only around the top 10 users to participate in the collaboration. Meanwhile, the communication cost can also be reduced if only a few users need to participate in the collaboration.

Fig. 6 shows the performance of the proposed SUM fusion performance versus the performance of AND fusion and OR fusion. For AND fusion, the final decision is set to be positive if all the users participating in the fusion report positive local decisions. In contrast, for OR fusion, the final decision is set to be positive if at least one user participating in the fusion report positive local decision. Since it is not beneficial to add all users in the fusion, in the simulations, only the top 5 and 10 users with the highest SNR are chosen to participate in the fusion in Fig. 6(a) and Fig. 6(b), respectively. Fig. 6(c) shows the comparisons for all users participating in the fusion. In general, the SUM fusion rule performs better than the other two fusion rules. Basically, the case where five users who have the highest SNR participate in the fusion has the highest detection performance. Adding more users is not helpful for the detection performance. In fact, adding more users could hurt the detection performance. Especially, the detection performance of the SUM fusion rule is not affected by the number of users participating in the fusion, but the

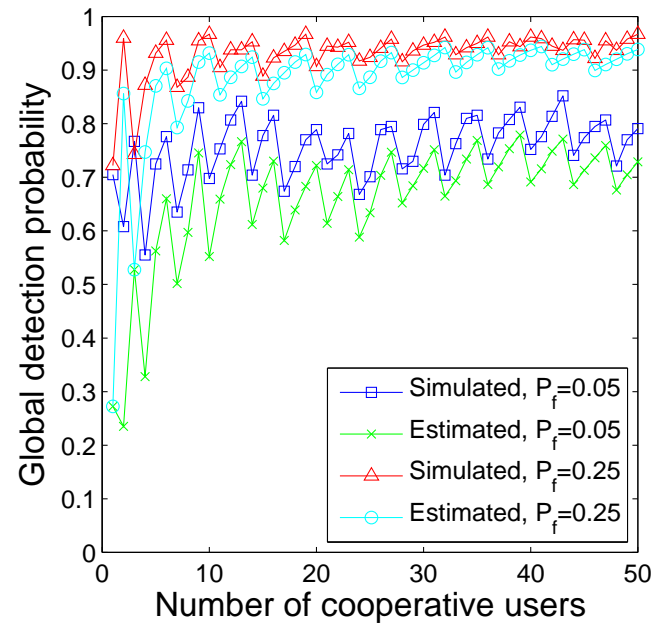

Fig. 5. The number of cooperative users v.s. global detection probability

performance degradation could be more serious if AND fusion rule is adopted.

\section{CONCLUSION}

This paper investigates the problem of collaborative sensing for the presence of the primary user in cognitive radio networks. Both the local and global decision making are based on CFAR detection mechanism. In particular, the distributions of measurements under both the hypotheses that the primary user is present and the primary user is absent are derived without making assumptions on any known signal distributions. The detection performance is also derived analytically based on the SUM fusion rule. From the simulation results, the estimated distributions of measurements match the true distributions well, and the derived detection performance is also very close to the simulation results. In particular, the detection performance of the proposed SUM fusion rule outperforms those of AND fusion and OR fusion. One important investigation result is that it is not necessary to have all secondary users within the communication region of a BS to participate in the fusion. It is better to have only a few users with the top SNR to participate in the fusion. In fact, adding more users in the fusion may not be helpful but hurt the detection performance. 


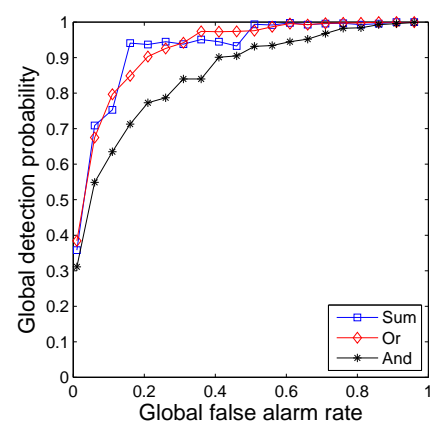

(a) SNR top 5

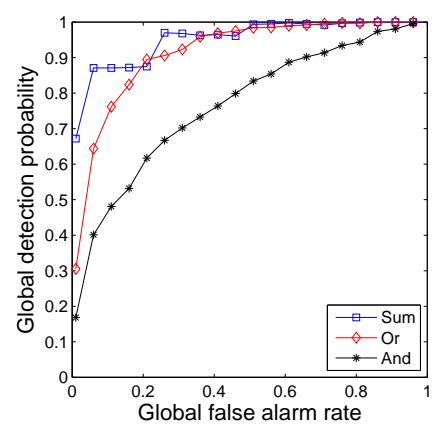

(b) SNR top 10

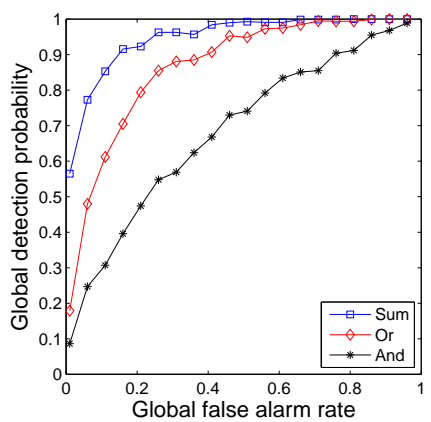

(c) All 50 users

Fig. 6. Comparison with AND and OR Fusion

This paper investigate the SUM fusion rule for collaborative sensing. Although it performs better than the AND fusion and OR fusion rules, other fusion rules may be worth to be explored in the future.

\section{ACKNOWLEDGMENT}

This work was supported in part by the Ministry of Science and Technology of Taiwan under grant MOST 103-2221-E-011 -095 .

\section{REFERENCES}

[1] B. Wang and K. R. Liu, "Advances in cognitive radio networks: A survey," IEEE Journal of Selected Topics in Signal Processing, vol. 5, no. 1, pp. 5-23, 2011.

[2] "IEEE 802.22-2011(TM) standard for cognitive wireless regional area networks (RAN) for operation in TV bands," July 2011.

[3] E. Peh and Y.-C. Liang, "Optimization for cooperative sensing in cognitive radio networks," in IEEE Wireless Communications and Networking Conference (WCNC), 2007, pp. 27-32.

[4] Federal Communications Commission, "Spectrum policy task force report, FCC 02-155," Nov 2002.

[5] H. V. Poor, An Introduction to Signal Detection and Estimation, 2nd ed Springer, 1998.

[6] E. Axell and E. G. Larsson, "Optimal and sub-optimal spectrum sensing of ofdm signals in known and unknown noise variance," IEEE Journal on Selected Areas in Communications, vol. 29, no. 2, pp. 290-304, 2011

[7] S. Enserink and D. Cochran, "A cyclostationary feature detector," in the Twenty-Eighth Asilomar Conference on Signals, Systems and Computers, vol. 2. IEEE, 1994, pp. 806-810.

[8] A. Sahai, N. Hoven, and R. Tandra, "Some fundamental limits on cognitive radio," in Allerton Conference on Communication, Control, and Computing. Monticello, Illinois, 2004, pp. 1662-1671.

[9] E. G. Larsson and M. Skoglund, "Cognitive radio in a frequencyplanned environment: some basic limits," IEEE Transactions on Wireless Communications, vol. 7, no. 12, pp. 4800-4806, 2008.

[10] A. Mariani, A. Giorgetti, and M. Chiani, "Snr wall for energy detection with noise power estimation," in IEEE International Conference on Communications (ICC). IEEE, 2011, pp. 1-6.

[11] R. Tandra and A. Sahai, "Snr walls for signal detection," IEEE Journal of Selected Topics in Signal Processing, vol. 2, no. 1, pp. 4-17, 2008
[12] S. Chaudhari, V. Koivunen, and H. V. Poor, "Autocorrelation-based decentralized sequential detection of ofdm signals in cognitive radios," IEEE Transactions on Signal Processing, vol. 57, no. 7, pp. 2690-2700, 2009.

[13] Z. Quan, W. Zhang, S. J. Shellhammer, and A. H. Sayed, "Optimal spectral feature detection for spectrum sensing at very low snr," IEEE Transactions on Communications, vol. 59, no. 1, pp. 201-212, 2011.

[14] J. Lundén, V. Koivunen, A. Huttunen, and H. V. Poor, "Collaborative cyclostationary spectrum sensing for cognitive radio systems," Signal Processing, IEEE Transactions on, vol. 57, no. 11, pp. 4182-4195, 2009.

[15] E. Axell, G. Leus, E. G. Larsson, and H. V. Poor, "Spectrum sensing for cognitive radio: State-of-the-art and recent advances," IEEE Signal Processing Magazine, vol. 29, no. 3, pp. 101-116, 2012.

[16] R. Fan and H. Jiang, "Optimal multi-channel cooperative sensing in cognitive radio networks," Wireless Communications, IEEE Transactions on, vol. 9, no. 3, pp. 1128-1138, 2010.

[17] S. Li, Z. Zheng, E. Ekici, and N. Shroff, "Maximizing system throughput by cooperative sensing in cognitive radio networks," Networking, IEEE/ACM Transactions on, vol. 22, no. 4, pp. 1245-1256, 2014.

[18] I. F. Akyildiz, B. F. Lo, and R. Balakrishnan, "Cooperative spectrum sensing in cognitive radio networks: A survey," Physical Communication, vol. 4, no. 1, pp. 40-62, 2011

[19] K. M. Thilina, K. W. Choi, N. Saquib, and E. Hossain, "Machine learning techniques for cooperative spectrum sensing in cognitive radio networks," Selected Areas in Communications, IEEE Journal on, vol. 31, no. 11, pp. 2209-2221, 2013.

[20] E. Peh and Y.-C. Liang, "Optimization for cooperative sensing in cognitive radio networks," in IEEE Wireless Communications and Networking Conference (WCNC). IEEE, 2007, pp. 27-32.

[21] Y. Selén, H. Tullberg, and J. Kronander, "Sensor selection for cooperative spectrum sensing," in 3rd IEEE Symposium on New Frontiers in Dynamic Spectrum Access Networks. IEEE, 2008, pp. 1-11.

[22] R. Chen, J.-M. Park, and K. Bian, "Robust distributed spectrum sensing in cognitive radio networks," in The 27th Conference on Computer Communications (INFOCOM). IEEE, 2008

[23] A. W. Min, K. G. Shin, and X. Hu, "Secure cooperative sensing in ieee 802.22 wrans using shadow fading correlation," IEEE transactions on Mobile Computing, vol. 10, no. 10, pp. 1434-1447, 2011.

[24] W. Ren, Q. Zhao, and A. Swami, "Power control in cognitive radio networks: how to cross a multi-lane highway," IEEE Journal on Selected Areas in Communications, vol. 27, no. 7, pp. 1283-1296, 2009.

[25] A. C. Davison, Statistical Models. Cambridge University Press, 2008

[26] P. Billingsley, Probability and Measure. John Wiley \& sons, 1995 